\section{Irinotecan beim kolorektalen Karzi- nom wichtiger Therapiebaustein}

Kontrollierte klinische Studien weisen darauf hin, dass Patienten mit metastasiertem kolorektalem Karzinom (mCRC) davon profitieren, wenn sie bereits als Erstlinientherapie eine Kombination aus Chemound Antikörpertherapie erhalten. Irinotecan ist ein wichtiger Baustein im Rahmen dieser modernen Therapiekonzepte. Mit den beiden derzeit zugelassenen monoklonalen Antikörpern Cetuximab bzw. Bevacizumab scheint sich die Wirksamkeit einer Kombinationstherapie mit Irinotecan zusätzlich zu verbessern. Dies führt dazu, dass mehr Patienten auf die Behandlung ansprechen und dann auch länger überleben. Laboruntersuchungen weisen auf synergistische Effekte zwischen Irinotecan und dem EGF-Rezeptor-Antikörper Cetuximab hin. Es zeigen sich erhöhte Konzentrationen von Irinotecan im Tumorgewebe, wenn Irinotecan gleichzeitig mit dem VEGF-Antikörper Bevacizumab gegeben wird. Beide Beobachtungen wurden inzwischen klinisch untermauert. In der sog. BOND-Studie, einer randomisierten Phase-III-Studie, bei Irinotecan-refraktären Patienten mit metastasiertem CRC war die Kombination Irinotecan/Cetuximab der alleinigen Cetuximab-Gabe auch nach einer Vorbehandlung mit Irinotecan überlegen: Die Patienten blieben im Median signifikant länger progressionsfrei (4,1 vs. 1,5 Monate; $\mathrm{p}<0,0001)$ und überlebten im Median knapp zwei Monate länger ( 8,6 vs. 6,9 Monate; $p=0,48)$. Erstmals konnte eine klinische Resistenz durch die Hinzunahme einer weiteren Substanz durchbrochen werden.
Irinotecan - Kombinationspartner für monoklonale Antikörper

Phase-II-Studien mit der Kombination Irinotecan/5-FU/FS plus Cetuximab als Erstlinientherapie bei Patienten mit metastasiertem CRC zeigen Ansprechraten von über $70 \%$. Bei bis zu $25 \%$ der Patienten mit zunächst inoperablen Lebermetastasen konnten die Metastasen im Anschluss an die Behandlung reseziert werden. Dies bedeutet für die Patienten - solange keine weiteren Metastasierungsherde vorliegen eine potentielle Heilungschance. Ein zweiter wichtiger Punkt ist, dass im Rahmen dieser klinischen Studien die Rate der primär progredienten Patienten nur bei $5-10 \%$ liegt im Vergleich zu bislang $20-25 \%$. In einer US-amerikanischen Phase-III-Studie erreichte die Kombination aus Irinotecan/5-FU/FS in Kombination mit Bevacizumab eine signifikante Verlängerung der medianen Überlebenszeit auf 20,3 Monate im Vergleich zu 15,6 Monaten unter alleiniger Irinotecan/ 5-FU/FS-Gabe $(\mathrm{p}=0,00004)$.

\section{Valide Datenbasis für Irinotecan}

Die hohe Wirksamkeit von Irinotecan zeigte sich bereits in früheren Studien, weshalb Irinotecan auch als erste neue Substanz für die Behandlung des metastasierten CRC zugelassen wurde. Die Kombinationstherapie mit Irinotecan plus 5-FU/FS (FOLFIRI) hatte erstmals nach jahrelanger therapeutischer Stagnation unter Phase-III-Studienbedingungen einen statistisch signifikanten Überlebensvorteil gegenüber der alten Standardtherapie (5-FU/FS) gezeigt (17,4 vs. 14,1 Monate; Douillard et al., Lancet 2000). Der statistisch signifikante Überlebensvorteil wurde in einer weiteren Phase-III-Studie bestätigt (Saltz et al., NEJM 2000). In einer PhaseIII-Studie erreichte Köhne mit dem FOLFIRI-Regime sogar eine mediane Überlebenszeit von über 20 Monaten (Köhne et al., ASCO 2003).

\section{Magenkarzinom - Irinotecan zeigt viel} versprechende Ergebnisse

Auch beim fortgeschrittenen Magenkarzinom erreicht Irinotecan in Kombination mit 5-FU/FS höhere Ansprechraten und tendenziell längere Überlebenszeiten als die bisher als Standard geltenden Cisplatin-haltigen Regime: In mehreren zum Teil randomisierten klinischen Studien, darunter auch eine Phase-III-Studie, liegen die Remissionsraten bei $40 \%$. Die Patienten bleiben im Median 5-6 Monate progressionsfrei und überleben im Median 9-11 Monate. Die Irinotecan-haltige Therapie ist ambulant durchführbar und wird im Vergleich zu den Cisplatin-haltigen Regimen subjektiv und objektiv von den Patienten wesentlich besser vertragen. Hierfür wird noch in 2005 die Zulassung angestrebt.

Weitere Informationen be Ogilvy Healthworld Düsseldorf

Bettina Strey

Postfach 190024

40110 Düsseldorf

Tel. +49 0211 49700-536

E-Mail bettina.strey@ogilvy.com

\title{
PharmaTicker+++ PharmaTicker+++ PharmaTicker+++ PharmaTicker+++
}

sanofi-aventis. Die europaweite Zulassung für Taxotere ${ }^{\circledR}$ (Docetaxel) in Kombination mit Doxorubicin und Cyclophosphamid zur adjuvanten Behandlung des operablen Mammakarzinoms mit Lymphknotenbefall und in Kombination mit Herceptin als Erstbehandlung für HER2/neu-positives metastasiertes Mammakarzinom ist erfolgt.

Aventis Pharma Deutschland $\mathrm{GmbH}$

Ein Unternehmen der sanofi-aventis Gruppe

Judith Kramer

Tel. +49 69 305-84412, Fax -84418

E-mail judith.kramer@aventis.com
Unter der Internetadresse www.hoffnung-beidarmkrebs.de finden Patienten, Angehörige und Interessierte ab sofort viele nützliche Informationen rund um das Thema Darmkrebs. Auf der Homepage (oder per Post) kann auch eine kostenlose Patientenbroschüre bestellt werden.

Weitere Informationen bei:

Aktionsbündnis «Hoffnung bei Darmkrebs»

Postfach 130120

50495 Köln essex pharma GmbH. Das pegylierte liposomale Doxorubicin Caelyx ${ }^{\circledR}$ gehört zu den Substanzen, die in den Leitlinien 2005 zur Diagnose und Therapie des Mammakarzinoms der Organkommission Mamma der Arbeitsgemeinschaft Gynäkologische Onkologie (AGO) als Mono- und auch Kombinationstherapie empfohlen werden.

Weitere Informationen be: essex pharma GmbH Dr. Detlef Hecker

Tel. +49 89627 31-277, Fax -253

E-mail detlef.hecker@essex.de

\begin{tabular}{ll}
\hline KARGER & ( 2005 S. Karger GmbH, Freiburg \\
& $\begin{array}{l}\text { Accessible online at: } \\
\text { Fax + 49 761452 } 0714\end{array}$ \\
$\begin{array}{l}\text { E-mail Information@Karger.de } \\
\text { www.karger.com }\end{array}$ & www.karger.com/onk \\
&
\end{tabular}




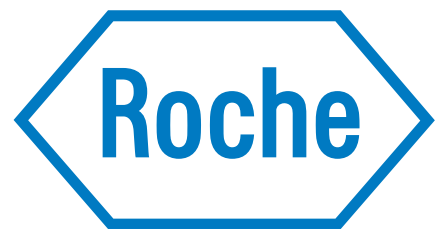

\section{Capecitabin (Xeloda ${ }^{\circledR}$ ) erhält positives Votum für die adjuvante Therapie des Kolonkarzinoms}

Das orale Chemotherapeutikum Capecitabin $\left(\right.$ Xeloda $\left.^{\circledR}\right)$ hat jetzt die Zulassungsempfehlung vom Komitee für Produkte der Humanmedizin der Europäischen Union (Committee for Human Medicinal Products/CHMP) für die adjuvante Therapie des Kolonkarzinoms erhalten. Mit einer vollständigen Marktzulassung in dieser neuen Indikation wird innerhalb der nächsten Wochen gerechnet.

Die positive Stellungnahme basiert auf den Ergebnissen der X-ACT-Studie (Xeloda in Adjuvant Colon Cancer Therapy). An der weltweit durchgeführten Phase-III-Studie waren 164 klinische Zentren beteiligt, die insgesamt 1987 Patienten in die Studie einbrachten. Die Patienten erhielten entweder 5-Fluorouracil/Leucovorin (5-FU/LV) als Bolus nach dem Mayo-Klinik-Schema oder das orale Fluoropyrimidin Capecitabin. Die Behandlung erfolgte über insgesamt 24 Wochen. Primärer Studienendpunkt war das krankheitsfreie Überleben.

Die Studienresultate zeigten, dass Capecitabin der Standardtherapie hinsichtlich der

Wirksamkeit und Sicherheit überlegen ist:

- Nach einer medianen Beobachtungszeit von 3,8 Jahren lebten noch $64,2 \%$ der Patienten aus der Capecitabin-Gruppe im Vergleich zu 60,6\% der Patienten aus der 5-FU/LV-Gruppe ohne erneute Krankheitsanzeichen $(\mathrm{p}=0,0528)$. Das Rückfallrisiko wurde um 14\% reduziert.

- Bezüglich des rezidivfreien Überlebens war Capecitabin dem Mayo-KlinikSchema signifikant überlegen (65,5 vs. $61,9 \% ; p=0,0407)$.
- Hinsichtlich des 3-Jahres-Gesamtüberlebens zeigte sich eine Gleichwertigkeit mit einer tendenziellen Überlegenheit von Capecitabin: $81,3 \%$ der Capecitabin-Patienten hatten im Vergleich zu $77,6 \%$ der nach dem Mayo-KlinikRegime behandelten Patienten überlebt ( $\mathrm{p}=0,0706)$.

- Die oral verabreichte Chemotherapie mit Capecitabin ist besser verträglich als die herkömmliche intravenöse Chemotherapie. Es kommt signifikant seltener zu Haarausfall, Diarrhoe, Stomatitis, Übelkeit/Erbrechen und Neutropenie $(\mathrm{p}<0,001)$.

- Patienten müssen das Krankenhaus bzw. die Praxis unter einer Therapie mit $\mathrm{Ca}$ pecitabin im Durchschnitt nur $8 \times$ gegenüber 30 Konsultationen unter einer Therapie mit 5-FU/LV aufsuchen.

Weitere Informationen bei:

Hoffmann-La Roche AG, Dr. med. H.-U. Jelitto 79639 Grenzach-Wyhlen, Deutschland

Tel. +49 7624 14-2400, Fax -3366

www.roche.de

\section{Major New Study Focuses on Best Treatment for Older Women with Receptor-Negative Early Breast Cancer}

The study, announced at the 9th International Conference «Primary Therapy of Early Breast Cancer», will investigate the effectiveness of a novel form of chemotherapy, called pegylated liposomal doxorubicin $\left(\right.$ Caelyx $^{\circledR}$, Schering-Plough Corporation), on breast cancer recurrence and survival after surgery. It will also com- pare Caelyx ${ }^{\circledR}$ with another, low-dose combination of anticancer drugs.

The CASA study (IBCSG 32-05 / BIG 1-05) will be conducted with 1296 patients aged 66 years or older with early breast cancer classified as nonresponsive to hormonal treatments. The duration of adjuvant treatment programs in the trial will be 16 weeks.

Nearly 1300 women aged 66 years and over with the type of early breast cancer that does not respond to hormone treatments, such as tamoxifen, will be recruited to the Chemotherapy Adjuvant Studies for Women at Advanced Age (CASA) trial. It will be coordinated by the International Breast Cancer Study Group (IBCSG), with worldwide participation by the Breast International Group (BIG) and support from Schering-Plough Corporation.

The trial, which will begin enrolling patients in the first half of 2005 , is expected to take approximately four and a half years to complete.

For further information please visit http://www.ibcsg.org.

\section{PharmaTicker+++ PharmaTicker+++ PharmaTicker+++ PharmaTicker+++}

Bayer HealthCare AG. Bayer Pharmaceuticals Corporation und Onyx Pharmaceuticals beginnen eine randomisierte Phase-III-Doppelblindstudie mit BAY 43-9006. Dabei wird BAY 43-0996 als Monowirkstoff bei Patienten mit fortgeschrittenem hepatozellulärem Karzinom (HCC, Leberkrebs) geprüft.

Weitere Informationen bei:

Bayer HealthCare AG, Helmut Schäfers

Tel. +49 214 30-58308

E-mail helmut.schaefers@bayerhealthcare.com
AstraZeneca. Mit dem reinen Östrogenrezeptor-Antagonisten Faslodex ${ }^{\circledR}$ (Fulvestrant) steht seit einem Jahr ein zusätzlicher endokriner Therapieschritt zur Verfügung. Seit der Zulassung wurde Faslodex ${ }^{\circledR}$ von mehreren Fachgesellschaften als zusätzliche Therapieoption anerkannt und empfohlen.

Weitere Informationen bei:

Publicis Vital PR, Natalie Fiebig

Tel. +49 221 912719-85, Fax -47

E-mail natalie.fiebig@publicis-vital-pr.de
Baxter Deutschland GmbH. Für das FOLFOXSchema sind die FOLFusoren von Baxter das Applikationssystem der Wahl. Die rein physikalische Pumpe sorgt für exakte und sichere Infusionsraten und ist somit hervorragend für die ambulante Therapie geeignet.

Weitere Informationen bei:

Baxter Deutschland $\mathrm{GmbH}$

Annette Berger

Tel. +49 89 31701-524

E-mail annette_berger@baxter.com

\begin{tabular}{ll}
\hline KARGER & @ 2005 S. Karger GmbH, Freiburg \\
& $\begin{array}{l}\text { Accessible online at: } \\
\text { www.karger.com/onk }\end{array}$ \\
$\begin{array}{l}\text { E-mail Information } @ \text { Karger.de } \\
\text { www.karger.com }\end{array}$ &
\end{tabular}

